\title{
Can a relativistic differential equation be set up to treat the angularity of the valence electron density in heavy atom clusters?
}

\author{
N.H. March ${ }^{\mathrm{a}, \mathrm{b}, \mathrm{c}}$, M.L. Glasser ${ }^{\mathrm{d}, \mathrm{e}, *}$ \\ a ICTP, Trieste, Italy \\ ${ }^{\mathrm{b}}$ Department of Physics, University of Antwerp, Antwerp, Belgium \\ c Oxford University, Oxford, England, United Kingdom \\ d Department of Theoretical Physics, University of Valladolid, Valladolid, Spain \\ e Department of Physics, Clarkson University, Potsdam, NY, USA
}

\section{A R T I C L E I N F O}

\section{Article history:}

Received 4 November 2014

Accepted 13 November 2014

Available online 18 November 2014

Communicated by V.M. Agranovich

\section{Keywords:}

Heavy atom cluster

Electron density

Relativistic kinetic energy

Differential equation

\begin{abstract}
A B S T R A C T
This work provides an explicit relativistic non-linear differential equation to estimate the ground-state electron density, and especially its directionality dependence, for large clusters of heavy atoms, such as $\mathrm{Pb}$, at their experimentally measured equilibrium geometry. The study embodies the early theory of Vallarta and Rosen, which seems to us to build a firm foundation on relativistic semi-classical many-electron theory. Assuming a finite nuclear radius for the heavy atoms would be advisable in subsequent numerical applications.
\end{abstract}

(C) 2014 Published by Elsevier B.V.

\section{Theory}

The earliest relativistic electron density theory goes back, at least, to Vallarta and Rosen [1]. These authors employed special relativity in the form of the relation between momentum and kinetic energy for an electron at the Fermi $(F)$ level to write the constant chemical potential $\mu$ throughout the entire inhomogeneous electron density distribution $n(\vec{r})$ as

$\mu=\left[c^{2} p_{F}^{2}(\vec{r})+m_{0}^{2} c^{4}\right]^{1 / 2}-m_{0} c^{2}+V(\vec{r})$,

where $V(\vec{r})$ is the self-consistent potential energy to be determined. The Fermi momentum $p_{F}(\vec{r})$ for, say, a cluster of atoms like $\mathrm{Pb}$ is related to $n(\vec{r})$ by phase space considerations [2], the result being

$n(\vec{r})=\frac{8 \pi}{3 h^{3}} p_{F}^{3}(\vec{r})$.

Eliminating $p_{F}(\vec{r})$ from (1) by means of (2) readily yields

$\mu=\left[B c^{2} n^{2 / 3}(\vec{r})+m_{0}^{2} c^{4}\right]^{1 / 2}-m_{0} c^{2}+V(\vec{r})$,

\footnotetext{
* Corresponding author at: Department of Physics, Clarkson University, Potsdam, NY, USA.

E-mail address: laryg@clarkson.edu (M.L. Glasser).
}

where $B=(3 / 8 \pi)^{2 / 3} h^{2}$. Adding the explicit requirement of selfconsistency, we have that $V(\vec{r})$ is also related to $n(\vec{r})$ by the electrostatic Poisson equation, namely

$\nabla^{2} V(\vec{r})=4 \pi n(\vec{r}) e^{2}$.

We next form $\nabla V(\vec{r})$ entering the left-hand side of Eq. (4) by applying the gradient operator with respect to $\vec{r}$ to both sides of (3). This readily leads to

$\nabla V(\vec{r})=-\left[\frac{1}{3} B c^{2} n^{-1 / 3}(\vec{r}) \nabla n(\vec{r})\right]\left[B c^{2} n^{2 / 3}(\vec{r})+m_{0}^{2} c^{4}\right]^{-1 / 2}$.

Inserting (5) into the left-hand side of (4) yields

$$
\begin{aligned}
-4 & \pi n(\vec{r}) e^{2} \\
= & \nabla \cdot\left\{\left[B c^{2} n^{2 / 3}(\vec{r})+m_{0}^{2} c^{4}\right]^{-1 / 2}\left[\frac{1}{3} B c^{2} n^{-1 / 3}(\vec{r}) \nabla n(\vec{r})\right]\right\} \\
& +\left[B c^{2} n^{2 / 3}(\vec{r})+m_{0}^{2} c^{4}\right]^{-1 / 2}\left\{\frac{1}{3} B c^{2} n^{-1 / 3}(\vec{r}) \nabla^{2} n(\vec{r})\right. \\
& \left.-\frac{1}{9} B c^{2} n^{-4 / 3}(\vec{r})[\nabla n(\vec{r})]^{2}\right\} .
\end{aligned}
$$

Though somewhat complicated, to our knowledge this is the first time a relativistic non-linear differential equation has been derived explicitly in a form which may well be useful relative to the experimental equilibrium geometry for describing some aspects of the angularity of $n(\vec{r})$ in large clusters of heavy atoms, such as $\mathrm{Pb}$. 
While Eq. (6) represents the main result of this letter, we wish to conclude by adding some brief comments in relation to linear response theory when the potential $V(\vec{r})$, but now assumed weak enough to allow one to work only to $O(V)$, is "switched on" to an originally uniform relativistic electron liquid of specified density $n_{0}$ [3]. Then one can generalize the non-relativistic linear response function $\vec{r}$-space theory of March and Murray [4] to read

$n(\vec{r})-n_{0}=\int F_{R}\left(\left|\vec{r}-\vec{r}_{0}\right|\right) V\left(\vec{r}_{0}\right) d \vec{r}_{0}$.

To date, the relativistic response function $F_{R}$ entering (7) is not known in closed analytic form in $\vec{r}$-space, whereas, as $c \rightarrow \infty, F$ in Eq. (7) is determined exactly by the first order spherical Bessel function $j_{1}$ [4]. Analytical progress in the relativistic case under discussion has proven tractable in $\vec{k}$ (Fourier transform) space [5].

Now, if we approximate the relativistic linear response formula (7) for the case of a slowly varying potential $V\left(\vec{r}_{0}\right)$ such that $V(\vec{r})$ is approximately equal to $V\left(\vec{r}_{0}\right)$, then we find

$n(\vec{r})-n_{0} \approx V(\vec{r}) \int F_{R}\left(\left|\vec{r}-\vec{r}_{0}\right|\right) d \vec{r}_{0}$
In the non-relativistic limit, $c \rightarrow \infty$ the last expression is approximately $\left(4 k_{F} / \pi e^{2} a_{0}\right) d V(\vec{r})$, where $k_{F}$ is the Fermi wave number, $a_{0}$ is the Bohr radius and $d$ is a dimensionless geometrical factor.

\section{Acknowledgements}

N.H.M. wishes to acknowledge that his contribution to this note was brought to fruition during a stay at the Abdus Salam ICTP, Trieste. Thanks are due to Prof. V.E. Kravtsov for a most stimulating visit and for kind hospitality. N.H.M. also thanks Prof. D. Lamoen and C. Van Alsenoy for making possible his continuing affiliation with the University of Antwerp. M.L.G. thanks Prof. L.M. Nieto and the University of Valladolid for continuing hospitality.

\section{References}

[1] M.S. Vallarta, N. Rosen, Phys. Rev. 41 (1932) 708.

[2] N.H. March, Electron Density Theory of Atoms and Molecules, Academic Press NY, 1992.

[3] N.H. March, Phys. Chem. Liq. 35 (1997) 59.

[4] N.H. March, A.M. Murray, Phys. Rev. 120 (1960) 830.

[5] B. Jancovici, Nuovo Cimento 25 (1962) 428. 